\title{
CALCULATION OF $H^{*}\left(B B O[k] ; Z_{2}\right)$
}

\section{ROBERT R. CLOUGH}

1. Introduction. Let $X[\mu]$ denote the $(\mu-1)$-connected covering of the space $X$. In [5], Stong calculated $H^{*}\left(B O[\mu] ; Z_{2}\right)$ as an algebra. $\mathrm{He}$ also partially determined the operation of the Steenrod algebra Q(2) on $H^{*}\left(B B O[\mu] ; Z_{2}\right)$. He used this result to show that certain manifolds are cobordic to zero. More recently, Gitler and Mahowald [1] and [2] have used Stong's calculations to help obtain results on geometric dimension and immersion of manifolds.

Stong's work leads us naturally to considering the highly connected coverings of other spaces involved in the Bott periodicity theorem. This amounts to calculating $H^{*}\left(\Omega^{\lambda}(B O[\mu]) ; Z_{2}\right)$. Of course, it is necessary only to carry out the calculations for $0 \leqq \lambda \leqq 7(\bmod 8)$.

Stong disposed of the case $\lambda=0(\bmod 8)$. In this paper, we shall treat the case $\lambda=7(\bmod 8)$. There are two alternative ways to look at the space $\Omega^{7}(B O[\mu])$. First, we can consider $\Omega^{7}(B O[9])=B B O$, the classifying space of $B O$ regarded as an $H$-space. In this context, $\Omega^{7}(B O[\mu+7])=B B O[\mu]$, and we are calculating the cohomology of the highly connected coverings of $B B O$. Second, we can recall that $B B O=S U / S O$ as a "homogeneous" space. In this paper, we choose to employ the notation $B B O$ because of its simplicity. However, the $S U / S O$ point of view is also useful. For example, $S U / S O$ is the fiber of the map $B S O \rightarrow B S U$ corresponding to "complexification." Thus we have a fibration

$$
\mathrm{BBO} \rightarrow \mathrm{BSO} \rightarrow \mathrm{BSU} .
$$

We shall denote by $H^{*}(X)$ the $Z_{2}$-cohomology of $X$; we shall use no other coefficient group. Let $Q_{k}=S q^{2}, S q^{2}, S q^{3}$, or $S q^{5}$ depending on whether $k=1,2,3$, or $5(\bmod 8)$ respectively. Let $G_{k}=\pi_{8 j+k}(B B O)$. Let $\theta_{j}$ denote the image in $H^{*}(B B O)$ of Stong's $\theta_{j}$ of $H^{*}(B S O)$ under the map $B B O \rightarrow B S O$ of the above fibration. Let $\phi(n)$ be the number of integers $s$ such that $0<s \leqq n$ and $s=0,1,2$, or $4(\bmod 8)$ and let $L(n)$ be one plus the number of ones in the dyadic expansion of $n-1$. Let $P\left[x_{1}, x_{2}, \cdots\right]$ and $E\left[x_{1}, x_{2}, \cdots\right]$ denote the $Z_{2}$ polynomial and exterior algebras generated by $\left\{x_{1}, x_{2}, \cdots\right\}$.

Theorem 1.

$$
H^{*}(B B O[8 j+k])=\frac{H^{*}\left(K\left(G_{k}, 8 j+k\right)\right)}{\operatorname{Ideal}\left[\alpha(2) Q_{k} b_{8 j+k}\right]} \otimes E\left[\theta_{i} \mid L(i) \leqq \phi(8 j+k)\right] .
$$

Received by the editors April 4, 1969. 
In $\$ 2$ we prove this theorem, along with a similar result for $H^{*}(U[2 j-1])$. Singer already has the latter result [4], but we need it in a slightly different form. We also write down an explicit calculation of $H^{*}\left(K\left(G_{k}, 8 j+k\right)\right) /$ Ideal $\left[Q(2) Q_{k} b_{8 j+k}\right]$ as a $Z_{2}$-algebra.

2. Details. By $A, B$, and $C$ we shall mean the sets of elements of $Q(2)$ of type $A, B$, and $C$ according to Stong's notation [5, p. 532]. Let $X(n)=\{\xi \in X \mid$ excess $(\xi) \leqq n-1\}$, where $X=A, B, C$, or $D$ and $D$ is defined below. Denote the elements of $A, B, C$, and $D$ by $\alpha, \beta$, $\gamma$, and $\delta$ respectively. For $\xi=\alpha, \beta$, or $\gamma$ we always have $\xi=I+\sum J$ where $I=\left(I_{0}, I_{1}\right)$. Stong writes down $I_{1}$ explicitly. For $\xi=\gamma$, we have $\sum J=0$. By $X^{\prime}(n)$ we mean $\{(\xi, 3) \mid \xi \in X(n)\}$. The notations $\alpha^{\prime}, \beta^{\prime}$, $\gamma^{\prime}$, and $I^{\prime}+\sum J^{\prime}$ then have the obvious meanings. For $\xi^{\prime}=\gamma^{\prime}$ we have $\sum J^{\prime}=0$.

Consider now the set $A(2 j-1)$ where $j$ is fixed. We wish to define $\hat{\alpha}$ for each $\alpha \in A(2 j-1)$ such that $\operatorname{rank}(\alpha) \geqq j-3$. Recall that rank $(\alpha)$ is the number of 2's in $I_{1}$ when $I_{1}$ is written in "brackets notation," i.e., $I_{1}=\left(a_{1}, \cdots, a_{n}\right)=\left[a_{1}-2 a_{2}, a_{2}-2 a_{3}, \cdots, a_{n-1}-2 a_{n}, a_{n}\right]$. The sum of the components of $I$, when $I$ is written in brackets notation is equal to excess $(I)$. If $I=[2 \cdots 2]$ where there are $j-32$ 's, then $\alpha=\alpha_{j-3}$ in Stong's notation. If $\operatorname{rank}(\alpha)=j-3$, let $\hat{\alpha}=\alpha$. Define $\hat{\alpha}_{j-2}=\left(2^{j-1}-2, \alpha_{j-3}\right)$ and $\hat{\alpha}_{j-1}=\left(2^{j}-2, \hat{\alpha}_{j-2}\right)$. Define $\hat{\alpha}$ for rank $(\alpha)$ $=j-2$ or $j-1$ by applying the same operations to $\hat{\alpha}_{j-2}$ and $\hat{\alpha}_{j-1}$ that Stong applies to $\alpha_{j-2}$ and $\alpha_{j-1}$ to get $\alpha$.

If $R$ is a ring and $X \subset R$, then Ideal $[X]$ is the left ideal generated by $X$. We frequently identify $S q^{I}$ with $I$ and $S q^{n}$ with $n$ to simplify notation. Thus, $Q(2) / \operatorname{Ideal}[1,3]$ means $Q(2)$ modulo the left ideal generated by $S q^{1}$ and $S q^{3}$. Clearly if we replace the $\alpha$ such that $j-1 \geqq$ $\operatorname{rank}(\alpha) \geqq j-3$ by $\hat{\alpha}$ we still have a basis of $Q(2) /$ Ideal $[1,3]$. Warning: The $\hat{\alpha}$ depend on which $j$ we fixed in the beginning to define them. Properly they should always bear a subscript. Since we shall always clearly fix the value of $j$, however, this will be unnecessary. Note that $\hat{I}=I$ for each $\hat{\alpha}$. Also $\hat{\alpha}=I+\sum \hat{J}$ where $\hat{J}>I$ in lexicographical order, but where the $\hat{J}$ may not correspond nicely to the $J$ in the $\sum J$ of $\alpha$. Let $E A(2 j-1)$ be the set of $\hat{\alpha}$.

TheOrem 2.

$$
H^{*}(U[2 j-1])=\frac{H^{*}(K(Z, 2 j-1))}{\operatorname{Ideal}\left[a(2) S q^{3} b_{2 j-1}\right]} \otimes P\left[\sigma \theta_{2 i} \mid L(2 i)>\phi(2 j)\right]
$$

where $\sigma$ is suspension in the universal fibration $U[2 j-1] \rightarrow * \rightarrow B U[2 j]$. Furthermore, 


$$
\begin{aligned}
& \frac{H^{*}(K(Z, 2 j-1))}{\operatorname{Ideal}\left[\hat{Q}(2) S q^{3} b_{2 j-1}\right]}=P\left[\xi b_{2 j-1} \mid \xi \in X(2 j-1)\right. \\
&\quad \text { for } X=A, B, \text { or } C \text { and } \quad \xi \neq \hat{\alpha}] \\
& \otimes E\left[\hat{\alpha} b_{2 j-1} \mid \hat{\alpha} \in E A(2 j-1)\right] .
\end{aligned}
$$

Proof. Let $H$ be the asserted value of $H^{*}(K(Z, 2 j-1)) /$ Ideal $\left[a(2) S q^{3} b_{2 j-1}\right]$. Let $E$ be the spectral sequence with fiber $H$ $\otimes P\left[\sigma \theta_{2 i} \mid L(2 i)>\phi(2 j)\right]$ and base $H^{*}(B U[2 j])$ with the obvious differentials. Because $\hat{I}_{0}=0$ for each $\hat{\alpha}$, and because $\left(\alpha_{j-3} b_{2 j-1}\right)^{2}$ $=\left(2^{j-1}+1, \alpha_{j-3}\right) b_{2 j-1}=\left(\alpha_{j-2}, 3\right) b_{2 j-1}=0$ so that all $\left(\hat{\alpha} b_{2 j-1}\right)^{2}=0$, we see that $E$ maps to the spectral sequence of $U[2 j-1] \rightarrow * \rightarrow B U[2 j]$ isomorphically. The proof of the theorem is now immediate. Q.E.D.

Now $Q(2)$ has a basis consisting of all of Stong's $\xi$ and $\xi^{\prime}$ together with all of the $(\xi, 1)$ and $\left(\xi^{\prime}, 1\right)$. We wish to single out certain of the $(\xi, 1)$ and $\left(\xi^{\prime}, 1\right)$ for use in our next result. Let $D(8 j+2)$ be the set of all $(\xi, 1)=(I, 1)+\sum(J, 1)$ and $\left(\xi^{\prime}, 1\right)=\left(I^{\prime}, 1\right)+\sum\left(J^{\prime}, 1\right)$ such that $I$ and $I^{\prime}$ have the form $\left[b_{1} \cdots b_{n} 0 \cdots 02\right]$ where $b_{n} \geqq 2$ and $n \geqq 0$. If $n$ is 0 , the tuple becomes $[0 \cdots 02]$. The $\delta=(\xi, 1)$ or $\left(\xi^{\prime}, 1\right)$ can be obtained from all $\xi$ except $\xi=\boldsymbol{\gamma}$ and $\xi=\gamma^{\prime}$.

THEOREM 3.

(1) $\frac{H^{*}(K(Z, 8 j+1))}{\operatorname{Ideal}\left[\propto(2) S q^{2} b_{8 j+1}\right]}=P\left[\gamma b_{8 j+1} \mid \gamma \in C(8 j+1)\right]$.

(2) $\frac{H^{*}\left(K\left(Z_{2}, 8 j+2\right)\right)}{\operatorname{Ideal}\left[\alpha(2) S q^{2} b_{8 j+2}\right]}=P\left[\gamma b_{8 j+2} \mid \gamma \in C(8 j+2)\right]$

$$
\otimes P\left[\delta b_{8+2} \mid \delta \in D(8 j+2)\right] \text {. }
$$

(3) $\frac{H^{*}\left(K\left(Z_{2}, 8 j+3\right)\right)}{\operatorname{Ideal}\left[\propto(2) S q^{3} b_{8 j+3}\right]}=\frac{H^{*}(K(Z, 8 j+3))}{\operatorname{Ideal}\left[Q(2) S q^{3} b_{8 j+3}\right]} \otimes \frac{H^{*}(K(Z, 8 j+4))}{\operatorname{Ideal}\left[\propto(2) S q^{5} b_{8 j+4}\right]}$.

(4) $\frac{H^{*}(K(Z, 8 j+5))}{\operatorname{Ideal}\left[Q(2) S q^{5} b_{8 j+5}\right]}=\frac{H^{*}(K(Z, 8 j+5))}{\operatorname{Ideal}\left[Q(2) S q^{3} b_{8 j+5}\right]} \otimes \frac{H^{*}(K(Z, 8 j+8))}{\operatorname{Ideal}\left[\propto(2) S q^{2} b_{8 j+8}\right]}$.

All the above isomorphisms are simultaneously isomorphisms of $Z_{2}$ algebras and $Q(2)$ modules if we agree that on the right sides of (3) and (4) we have $S q^{1} b_{8 j+3}=b_{8 j+4}$ and $S q^{3} b_{8 j+5}=b_{8 j+8}$.

We appeal to the following fibrations of Bott periodicity:

$$
\begin{aligned}
& U[8 j+1] \rightarrow B B O[8 j+1] \rightarrow B O[8 j+1] \\
& U[8 j+3] \rightarrow B B O[8 j+2] \rightarrow B O[8 j+2] \\
& U[8 j+3] \rightarrow B B O[8 j+3] \rightarrow B O[8 j+4] \\
& U[8 j+5] \rightarrow B B O[8 j+5] \rightarrow B O[8 j+8]
\end{aligned}
$$


These fibrations have the following transgressions respectively: $\tau b_{8 j+1}=S q^{1} b_{8 j+1} ; \tau b_{8 j+3}=S q^{2} b_{8 j+2} ; \tau b_{8 j+3}=0 ; \tau b_{8 j+5}=0$. In all fibrations, $\tau\left(\sigma \theta_{2 i}\right)=\left(\theta_{i}\right)^{2}$.

To prove the first parts of Theorems 1 and 3 we investigate the spectral sequence of the first fibration. Since $\tau b_{8 j+1}=S q^{1} b_{8 j+1}$, transgression "decreases excess by one" when applied to the $\xi b_{8 j+1}$. We deduce from Milnor-Moore [3, Proposition 4.3] that the map

$$
\frac{Q(2)}{\operatorname{Ideal}[1,3]} \stackrel{\cdot S q^{1}}{\longrightarrow} \frac{a(2)}{\operatorname{Ideal}[2]}
$$

is a monomorphism. By Stong's Proposition (p. 532), all of the $\xi, \xi^{\prime}$, $(\xi, 1)$ and $\left(\xi^{\prime}, 1\right)$ in $Q(2)$ except the $\gamma$ are equal to some $(\xi, 2),\left(\xi^{\prime}, 2\right)$, $(\xi, 1,2)$, or $\left(\xi^{\prime}, 1,2\right)$ modulo elements of higher lexicographical order. In particular, $\xi^{\prime}=(\xi, 3)=(\xi, 1,2)$ and the result is even more obvious for $\xi=\alpha, \beta, \alpha^{\prime}, \beta^{\prime}$, and $\gamma^{\prime}$. The other cases follow if we notice that $([\cdots 0 \cdots 002], 2)=([\cdots 0 \cdots 04],(3,1))$ together with the fact that $([\cdots 20 \cdots 04], 3)=[\cdots 10 \cdots 002]=\gamma^{\prime}$ and $([\cdots 10 \cdots 04], 3)=0$. Recall that $\sum J$ and $\sum J^{\prime}$ are both 0 for $\gamma$ and $\gamma^{\prime}$. Thus, the $E^{\infty}$ term of the spectral sequence of the first Bott fibration is $P\left[\gamma b_{8 j+1} \mid \gamma \in C(8 j+1)\right]$. The obvious maps

$$
\frac{H^{*}(K(Z, 8 j+1))}{\operatorname{Ideal}\left[\propto(2) S q^{2} b_{8 j+1}\right]} \leftrightarrow P\left[\gamma b_{8 j+1} \mid \gamma \in C(8 j+1)\right]
$$

must therefore be isomorphisms of $Z_{2}$-algebras and $a(2)$ modules.

The proof of the second parts of Theorems 1 and 3 is similar to the proof of the first part and employs the second Bott fibration. In this case, we use the monomorphism

$$
\frac{a(2)}{\operatorname{Ideal}[1,3]} \stackrel{S q^{2}}{\rightarrow} \frac{a(2)}{\operatorname{Ideal}[3]}
$$

which decreases excess by 2 . We use the methods of the previous paragraph to show that all of the $\xi, \xi^{\prime},(\xi, 1)$, and $\left(\xi^{\prime}, 1\right)$ except the $\gamma$ and $\delta$ are of the form $(\xi, 3)$ or $\left(\xi^{\prime}, 3\right)$ modulo elements of higher lexicographical order.

To handle the third and fourth parts of Theorems 1 and 3 we use the third and fourth Bott fibrations above. The only nontrivial part is to show that the exterior generators of the fiber which survive to $E^{\infty}$ actually square to 0 in $H^{*}(U[8 j+k])$ for $k=3$ and 5 . We shall first handle the case $k=3$. We proceed to alter the $\alpha$ once again. First let $\alpha=\alpha_{2(4 j+2)-3}$. In $\alpha(2) /$ Ideal[1], we know that $\left(2^{m-1}+1, \alpha\right)$ $=\left(\alpha_{m-2}, 3\right)$ where $m=(4 j+2)$. Thus, in $Q(2)$ we know that $\left(2^{m-1}+1, \alpha\right)$ 
$+\sum K=\left(\alpha_{m-2}, 3\right)$ where each $K$ is divisible on the right by $S q^{1}$. Using techniques similar to those of the first paragraph of this proof, we can routinely show that each $K>\left(2^{m}, I\right)$ in lexicographical order, where $I$ corresponds to $\alpha$. Define $\bar{\alpha}=\alpha+\sum \bar{K}$ where the $\bar{K}$ are the $\left(a_{2}, \cdots, a_{n}\right)$ such that $K=\left(a_{1}, \cdots, a_{n}\right)$ and $a_{1}=2^{m-1}+1$. Then $\bar{K}>\alpha$ in lexicographical order and, since the excesses of all the other $K$ are $>2 m-1$ we know that $\left(\bar{\alpha} b_{2 m-1}\right)^{2}=\left(\alpha_{m-2}, 3\right) b_{2 m-1}=0$. Form all other $\bar{\alpha}$ of rank $\geqq m-3$ just as we earlier formed the $\hat{\alpha}$.

The proof of the fourth parts of Theorems 1 and 3 is entirely similar. Q.E.D.

\section{REFERENCES}

1. S. Gitler and M. Mahowald, The geometric dimension of real stable vector bundles, Bol. Soc. Mat. Mexicana (2) 11 (1966), 85-107. MR 37 \#6922.

2. - The immersion of manifolds, Bull. Amer. Math. Soc. 73 (1967), 696700. MR $35 \# 4940$.

3. J. W. Milnor and J. C. Moore, On the structure of Hopf algebras, Ann. of Math. (2) 81 (1965), 211-264. MR 30 \#4259.

4. W. M. Singer, Connective fiberings over BU and $U$, Topology 7 (1968), 271-303. MR $38 \# 717$.

5. R. E. Stong, Determination of $H^{*}\left(B O(k, \cdots, \infty) Z^{2}\right)$ and $H^{*}(B U(k, \cdots, \infty)$, $\left.Z_{2}\right)$, Trans. Amer. Math. Soc. 107 (1963), 526-544. MR 27 \#1944.

University of Notre Dame 